BMJ Open

Diabetes

Research

\& Care

\title{
Superior weight loss with once-weekly semaglutide versus other glucagon-like peptide-1 receptor agonists is independent of gastrointestinal adverse events
}

\author{
Ildiko Lingvay (i) , ${ }^{1}$ Thomas Hansen, ${ }^{2}$ Stanislava Macura, ${ }^{2}$ Michel Marre, ${ }^{3,4}$ \\ Michael A Nauck, ${ }^{5}$ Raymond de la Rosa, ${ }^{6}$ Vincent Woo, ${ }^{7}$ Emre Yildirim, ${ }^{2}$ \\ John Wilding (10 ${ }^{8}$
}

To cite: Lingvay I, Hansen T, Macura S, et al. Superior weight loss with onceweekly semaglutide versus other glucagonlike peptide-1 receptor agonists is independent of gastrointestinal adverse events. BMJ Open Diab Res Care 2020;8:e001706. doi:10.1136/ bmjdrc-2020-001706

- Supplemental material is published online only. To view, please visit the journal online (http://dx.doi.org/10.1136/ bmjdrc-2020-001706).

Received 19 June 2020 Revised 8 September 2020 Accepted 8 September 2020

Check for updates

(C) Author(s) (or their employer(s)) 2020. Re-use permitted under CC BY-NC. No commercial re-use. See rights and permissions. Published by BMJ.

For numbered affiliations see end of article.

Correspondence to Dr Ildiko Lingvay; Ildiko.Lingvay@ UTSouthwestern.edu

\section{ABSTRACT}

Introduction Gastrointestinal (GI) adverse events (AEs) are the most common AEs with glucagon-like peptide-1 receptor agonists (GLP-1RAs). Weight loss (WL) is slightly greater in people who experience Gl AEs than those who do not. A previous mediation analysis of the SUSTAIN 1-5 trials indicated minor contribution of nausea/vomiting to the greater WL with once-weekly semaglutide versus comparators. Semaglutide demonstrated superior glycated hemoglobin and body weight (BW) reductions versus other GLP-1RAs in SUSTAIN 3 (versus exenatide extended release $2.0 \mathrm{mg}$ ), SUSTAIN 7 (versus dulaglutide) and SUSTAIN 10 (liraglutide $1.2 \mathrm{mg}$ ). The objective of this analysis was to assess if significantly greater WL with semaglutide versus other GLP-1RAs is mediated by nausea/vomiting and other GI AEs (diarrhea, constipation, dyspepsia) during dose escalation (baseline to week 12, when GI AEs are generally most prevalent) and from baseline to end of treatment (EOT: week 56 (SUSTAIN 3), 40 (SUSTAIN 7) or 30 (SUSTAIN 10)).

Research design and methods Subjects within trials were subdivided into those who reported (yes/no) nausea/ vomiting or any other GI AE. Change from baseline in BW was assessed within each trial and subgroup. A mediation analysis separated WL into direct or indirect (mediated by GI AEs) effects.

Results From baseline to week 12 or EOT, the nausea/ vomiting-mediated difference in WL was, respectively: 0.05 or $0.09 \mathrm{~kg}$ of $3.78 \mathrm{~kg}$ at EOT (SUSTAIN 3); 0.06 or $0.03 \mathrm{~kg}$ of $2.26 \mathrm{~kg}$ at EOT (low-dose comparison) and 0.08 or 0.04 $\mathrm{kg}$ of $3.55 \mathrm{~kg}$ at EOT (high-dose comparison) (SUSTAIN 7) and 0.05 or $0.09 \mathrm{~kg}$ of $3.82 \mathrm{~kg}$ at EOT (SUSTAIN 10). Conclusions In SUSTAIN 3, 7 and 10, nausea/vomiting by week 12 (end of dose escalation) or throughout treatment contributed minimally $(<0.1 \mathrm{~kg})$ to the superior WL with semaglutide versus GLP-1RA comparators at EOT.

\section{INTRODUCTION}

The association between type 2 diabetes (T2D) and overweight/obesity is well established, ${ }^{12}$ with more than $90 \%$ of people with T2D being overweight. ${ }^{3}$ Individuals with T2D and overweight/obesity are at increased risk of developing T2D complications compared

\section{Significance of this study}

What is already known about this subject?

- A previous mediation analysis, which evaluated the effect of nausea/vomiting on weight loss in the SUSTAIN 1-5 trials, showed that nausea/vomiting contributed only minimally to the superior weight loss with once-weekly semaglutide, a glucagonlike peptide-1 receptor agonist, versus mixed-class comparators.

What are the new findings?

- In this mediation analysis, we investigated the effect of nausea/vomiting within the glucagon-like peptide-1 receptor agonist (GLP-1RA) class (semaglutide versus other GLP-1RAs), which is known for its common but transient gastrointestinal (Gl) adverse events (AEs)

- Nausea/vomiting contributed minimally to the significantly greater body weight (BW) reductions with semaglutide versus exenatide extended release (SUSTAIN 3; 56 weeks), dulaglutide (SUSTAIN 7; 40 weeks) or liraglutide (SUSTAIN 10; 30 weeks); these reductions were predominantly independent of the Gl AEs of nausea, vomiting, diarrhea, dyspepsia and constipation.

- From baseline to week 12 (end of dose escalation) or to end of treatment (EOT), the nausea-/vomitingmediated difference in BW loss was, respectively: $0.05 \mathrm{~kg}$ or $0.09 \mathrm{~kg}$ of $3.78 \mathrm{~kg}$ seen at EOT (SUSTAIN 3); $0.06 \mathrm{~kg}$ or $0.03 \mathrm{~kg}$ of $2.26 \mathrm{~kg}$ seen at EOT (lowdose comparison) and $0.08 \mathrm{~kg}$ or $0.04 \mathrm{~kg}$ of $3.55 \mathrm{~kg}$ seen at EOT high-dose comparison) (SUSTAIN 7) and $0.05 \mathrm{~kg}$ or $0.09 \mathrm{~kg}$ of $3.82 \mathrm{~kg}$ seen at EOT (SUSTAIN 10).

- Similarly, a minimal amount of the greater weight loss at EOT observed with semaglutide versus other GLP-1RAs was mediated by the individual Gl AEs of nausea, vomiting, diarrhea, dyspepsia or constipation reported from baseline to week 12 and from baseline to EOT.

with people who are not overweight/obese. Body weight (BW) reductions of $\geq 5 \%$ improve glycemic control, lipid levels and 


\section{Significance of this study}

How might these results change the focus of research or clinical practice?

- The results of this analysis indicate that the superior weight loss observed with semaglutide versus GLP-1RA class comparators is mostly independent of Gl AEs, the most common AEs in this class. These results are consistent with the previous findings in SUSTAIN $1-5$ trials.

blood pressure. ${ }^{5} \mathrm{BW}$ control is an important component of an individualized, multifactorial approach to T2D management, as recommended in current treatment guidelines. ${ }^{6} 7$

Glucagon-like peptide-1 receptor agonists (GLP-1RAs) are recommended as second-line therapy (add-on to metformin) where minimizing weight gain, promoting weight loss or when hypoglycemia and cardiovascular risk reduction are considerations. ${ }^{7-9}$ All available GLP-1RAs (dulaglutide, exenatide, liraglutide and lixisenatide) have demonstrated weight loss in people with T2D. ${ }^{10-12}$ Semaglutide (Novo Nordisk, Denmark) is a GLP-1RA approved for the treatment of T2D as once-weekly (OW) subcutaneous ${ }^{13}$ and once-daily oral formulations. ${ }^{14}$ The efficacy and safety of OW semaglutide have been established in the global phase 3 SUSTAIN clinical trial program, encompassing subjects from across the continuum of T2D care. ${ }^{15-24}$ In addition to significantly greater reductions in glycated hemoglobin $\left(\mathrm{HbA}_{1 \mathrm{c}}\right)$, semaglutide demonstrated superior reductions in BW versus all comparators across all SUSTAIN trials. ${ }^{15-24}$

The SUSTAIN 3, 7 and 10 trials compared semaglutide with the GLP-1RAs OW exenatide extended release (exenatide ER), OW dulaglutide and once-daily liraglutide, respectively. In these trials, mean BW loss was significantly greater with semaglutide versus comparators at end of treatment (EOT: weeks 56, 40 and 30 for SUSTAIN 3, 7 and 10, respectively): SUSTAIN 3: $-5.6 \mathrm{~kg}$ vs $-1.9 \mathrm{~kg}$ with semaglutide $1.0 \mathrm{mg}$ vs exenatide ER 2.0 mg; SUSTAIN 7: $-4.6 \mathrm{~kg}$ vs $-2.3 \mathrm{~kg}$ with semaglutide 0.5 $\mathrm{mg}$ vs dulaglutide $0.75 \mathrm{mg}$ and $-6.5 \mathrm{~kg}$ vs $-3.0 \mathrm{~kg}$ with semaglutide $1.0 \mathrm{mg}$ vs dulaglutide $1.5 \mathrm{mg}$; SUSTAIN 10 : $-5.8 \mathrm{~kg}$ vs $-1.9 \mathrm{~kg}$ with semaglutide $1.0 \mathrm{mg}$ vs liraglutide $1.2 \mathrm{mg}$; all $\mathrm{p}<0.0001$. $^{172124}$

Consistent with the GLP-1RA class, ${ }^{25-27}$ gastrointestinal (GI) adverse events (AEs) were the most frequently reported AEs in the SUSTAIN 3, 7 and 10 trials: $42 \%$ with semaglutide $1.0 \mathrm{mg}$ vs $33 \%$ with exenatide ER 2.0 $\mathrm{mg}$ in SUSTAIN 3; $43 \%$ with semaglutide $0.5 \mathrm{mg}$ vs $33 \%$ with dulaglutide $0.75 \mathrm{mg}$ and $44 \%$ with semaglutide 1.0 $\mathrm{mg}$ vs $48 \%$ with dulaglutide $1.5 \mathrm{mg}$ in SUSTAIN 7; $44 \%$ with semaglutide $1.0 \mathrm{mg}$ vs $38 \%$ with liraglutide $1.2 \mathrm{mg}$ in SUSTAIN 10. ${ }^{172124}$ The five most commonly reported GI AEs in SUSTAIN 3, 7 and 10 were: nausea (23\% with semaglutide $0.5 \mathrm{mg}, 21 \%-22 \%$ with semaglutide $1.0 \mathrm{mg}$ and 12\%-20\% with comparators); vomiting (10\% with semaglutide $0.5 \mathrm{mg}, 7 \%-10 \%$ with semaglutide $1.0 \mathrm{mg}$ and $4 \%-10 \%$ with comparators); diarrhea (14\% with semaglutide $0.5 \mathrm{mg}, 11 \%-16 \%$ with semaglutide $1.0 \mathrm{mg}$ and $8 \%-18 \%$ with comparators); dyspepsia (3\% with semaglutide $0.5 \mathrm{mg}, 4 \%-7 \%$ with semaglutide $1.0 \mathrm{mg}$ and $3 \%-5 \%$ with comparators) and constipation (5\% with semaglutide $0.5 \mathrm{mg}, 5 \%-6 \%$ with semaglutide 1.0 $\mathrm{mg}$ and 3\%-5\% with comparators). ${ }^{17212428}$

Given the clinical significance of weight loss in T2D management, it is important to understand the mechanism by which semaglutide provides greater weight loss versus class comparators and, in particular, whether it is mediated by GI AEs. A previous mediation analysis examining superior weight loss with semaglutide versus mixed class comparators by GI AEs in the SUSTAIN 1-5 trials showed that only $0.07 \mathrm{~kg}$ of $2.3 \mathrm{~kg}$ (semaglutide $0.5 \mathrm{mg}$ ) and $0.5 \mathrm{~kg}$ of $6.3 \mathrm{~kg}$ (semaglutide $1.0 \mathrm{mg}$ ) of the treatment difference in weight loss was mediated by nausea/ vomiting. ${ }^{29}$

To further determine if GI AEs of nausea/vomiting and others are associated with weight loss, we performed a post hoc mediation analysis to examine the extent to which the treatment difference with semaglutide versus the other GLP-1RAs in the SUSTAIN 3, 7 and 10 trials might be driven by a difference in GI AEs (indirect effects) or treatment (direct effect). Data on nausea and/or vomiting were pooled and data on nausea, vomiting, diarrhea, constipation and dyspepsia were analyzed individually.

\section{MATERIALS AND METHODS}

\section{SUSTAIN 3, 7 and 10 trial designs}

The designs of the SUSTAIN 3, 7 and 10 trials have been previously published. ${ }^{172124}$ Briefly, subjects with inadequately controlled T2D were randomized to receive: (1) in SUSTAIN 3, semaglutide $1.0 \mathrm{mg}$ or exenatide ER $2.0 \mathrm{mg}$, in addition to existing oral antidiabetes drugs, over 56 weeks; ${ }^{17}$ (2) in SUSTAIN 7, semaglutide $0.5 \mathrm{mg}$ or $1.0 \mathrm{mg}$, or dulaglutide $0.75 \mathrm{mg}$ or $1.5 \mathrm{mg}$ in addition to metformin monotherapy, over 40 weeks; ${ }^{21}$ (3) in SUSTAIN 10 , semaglutide $1.0 \mathrm{mg}$ or liraglutide $1.2 \mathrm{mg}$, in addition to $1-3$ oral antidiabetes drugs, over 30 weeks. ${ }^{24}$

Semaglutide-treated subjects followed a fixed doseescalation regimen: ${ }^{1721} 24$ the $0.5 \mathrm{mg}$ maintenance dose was reached after 4 weeks of $0.25 \mathrm{mg} \mathrm{OW}$ and the 1.0 mg maintenance dose was reached after 4 weeks of 0.25 $\mathrm{mg}$ OW, followed by 4 weeks of $0.5 \mathrm{mg}$ OW. Exenatide ER was administered in accordance with its prescribing information $^{30}$ (ie, no dose escalation) and dulaglutide was administered in accordance with its phase III clinical trial program (ie, no dose escalation). ${ }^{31}$ The liraglutide $1.2 \mathrm{mg}$ maintenance dose was reached after 1 week of 0.6 mg once daily. ${ }^{24}$

For all three trials, prior to trial initiation, the protocol, the consent form and the subject information sheet were reviewed and approved according to local regulations by appropriate health authorities and by an independent ethics committee/institutional review board. Written informed consent was obtained from all participants. 
Post hoc analyses

Subjects in the SUSTAIN 3, 7 and 10 trials were subdivided according to whether or not they had reported either nausea or vomiting or both nausea and vomiting (nausea/vomiting), regardless of severity or duration. In addition, the subjects were subdivided according to whether or not they had reported any of the five most common GI AEs associated with semaglutide (nausea, vomiting, diarrhea, constipation or dyspepsia).

\section{Change from baseline in BW by GI AEs}

The change in BW from baseline to EOT (week 56 for SUSTAIN 3; week 40 for SUSTAIN 7; week 30 for SUSTAIN 10) in subjects who experienced GI AEs versus those who did not experience GI AEs was estimated from a mixed model for repeated measurements. The effect of GI AEs on the change from baseline in BW at EOT was compared in subjects with versus without GI AEs from baseline to week 12 (when GI AEs were found to peak and decline thereafter) and from baseline to EOT. The effect on the change in BW was analyzed by each of the five common GI AEs (nausea, vomiting, diarrhea, constipation or dyspepsia) individually and by nausea/ vomiting. Analyses for BW change were performed on the full analysis set. Subjects who discontinued treatment/initiated rescue medication contributed to the analysis based on the data observed prior to their discontinuation of treatment or initiation of rescue medication.

\section{Mediation analysis}

As with the previous analysis, ${ }^{29}$ a mediation analysis was performed to separate the overall effect of the GLP-1RAs on BW into direct or indirect (mediated by nausea or vomiting) effects, estimated using natural effect models with imputationbased estimation. ${ }^{32}$ Missing BW data were imputed using observed data within the same treatment group assuming that data were missing at random. The question assessed by the direct effect was: what is the effect of changing the treatment from comparator to semaglutide while maintaining the mediator at a value observed in the comparator arm? Conversely, the question assessed by the indirect effect was: what is the effect of changing the level of mediator between semaglutide and comparator (exenatide ER, dulaglutide or liraglutide)? As some of these factors are counterfactual (ie, things that did not occur but were possible) and nonobservable, a model was required to obtain estimates of the direct and indirect effects. The natural effect model for the estimation of direct and indirect effects included the interaction between treatment and GI AEs together with the baseline variables of BW and country as main effects, assuming no interaction between natural effects and baseline variables; standard errors of treatment differences were estimated by the bootstrap method. The model used to impute counterfactual values of BW also included the interaction between treatment and each baseline variable and the interaction between any $\mathrm{GI} \mathrm{AE}$ and each baseline variable.

\section{RESULTS}

The presented results of the SUSTAIN 3, 7 and 10 trials focus on the category of subjects with/without nausea/ vomiting, regardless of severity or duration (table 1; figures 1 and 2). The results, according to the common individual GI AEs associated with semaglutide (nausea, vomiting, diarrhea, constipation or dyspepsia), are provided in detail in the online supplemental material 1.

\section{Subject disposition and baseline characteristics by nausea/ vomiting}

Overall baseline characteristics, which have been previously published, were broadly similar between the three trials, with the exception of a longer diabetes duration in subjects in SUSTAIN 3 and SUSTAIN 10 versus SUSTAIN 7 (minimum/maximum of the mean across treatment groups: 9.0-9.4 years and 8.9-9.6 years vs 7.0-7.7 years, respectively).${ }^{1721}{ }^{24}$ Greater proportions of subjects with nausea/vomiting (occurring from baseline to week 12 and from baseline to EOT) discontinued treatment than subjects without. Subjects with nausea/vomiting generally had lower baseline BW than subjects without. There were no other differences in baseline characteristics for subjects with or without nausea/vomiting (table 1).

\section{Change from baseline in body weight in subjects with and without nausea/vomiting}

BW reductions with all four GLP-1RAs were consistently greater in subjects who experienced nausea/vomiting than in those who did not, and reductions with semaglutide were consistently greater than those seen with exenatide ER, dulaglutide or liraglutide, regardless of nausea/vomiting (figure 1).

\section{SUSTAIN 3 (semaglutide versus exenatide ER)}

At EOT, a weight change of $-7.0 \mathrm{~kg}$ was observed in subjects treated with semaglutide $1.0 \mathrm{mg}$ experiencing nausea/vomiting from baseline to week $12 \mathrm{vs}-5.3 \mathrm{~kg}$ in those who did not experience these events $(p=0.0274)$. The corresponding values for exenatide ER were -2.5 vs $-1.8 \mathrm{~kg}(\mathrm{p}=0.4322$; figure $1 \mathrm{~A})$.

In subjects treated with semaglutide $1.0 \mathrm{mg}$ experiencing nausea/vomiting at any time from baseline to EOT, a weight change of $-6.8 \mathrm{~kg}$ vs $-5.3 \mathrm{~kg}$ at EOT was observed versus those who did not experience these events $(p=0.0447)$. The corresponding values for exenatide ER were -3.3 vs $-1.6 \mathrm{~kg}(\mathrm{p}=0.0632)$.

Estimated treatment differences (ETDs) (95\% CIs) favored semaglutide versus exenatide ER in all comparisons (figure 1A).

\section{SUSTAIN 7 (semaglutide versus dulaglutide)}

At EOT, the weight change in subjects experiencing nausea/vomiting from baseline to week 12 versus those who did not experience these events was -5.5 


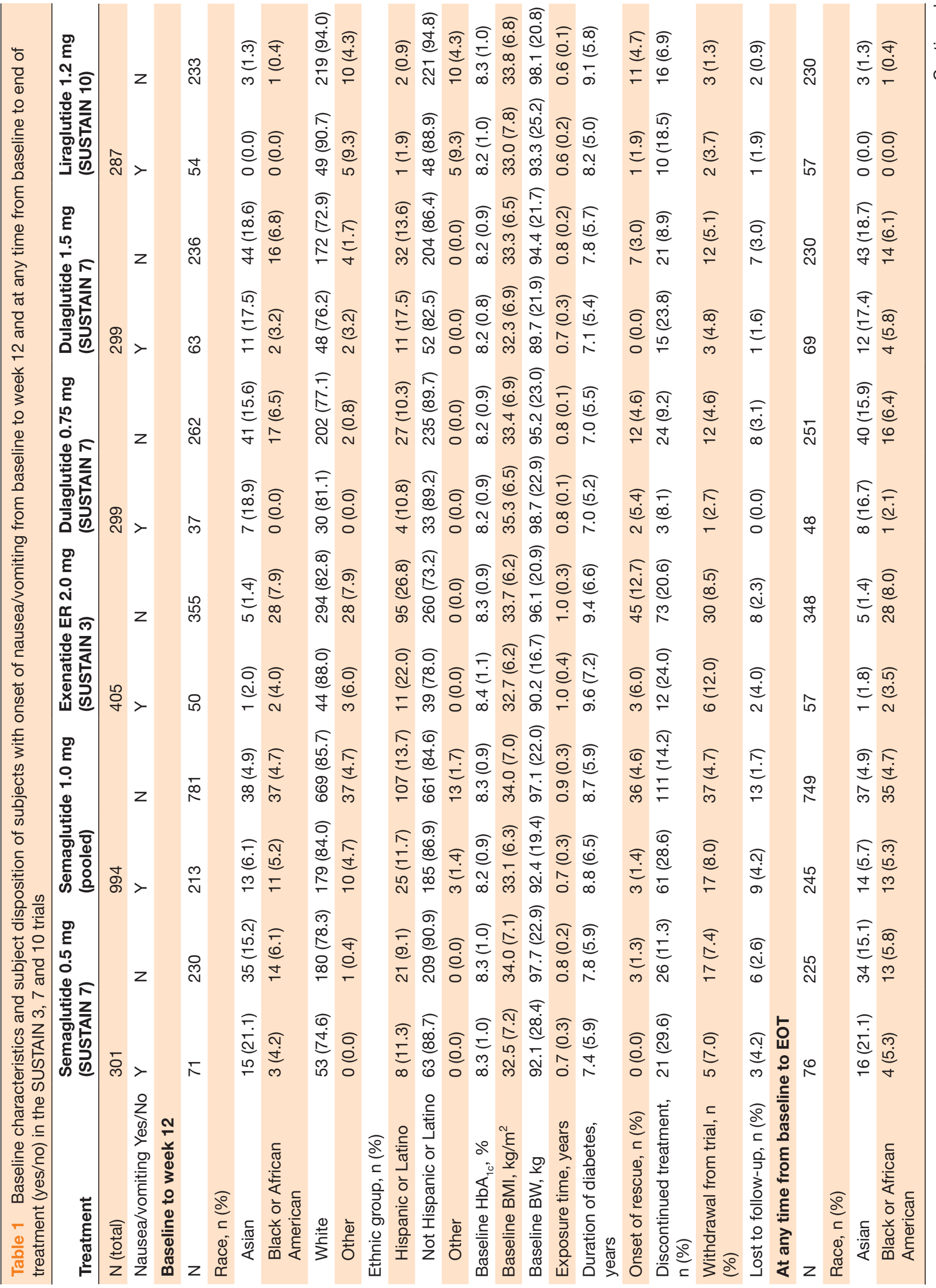




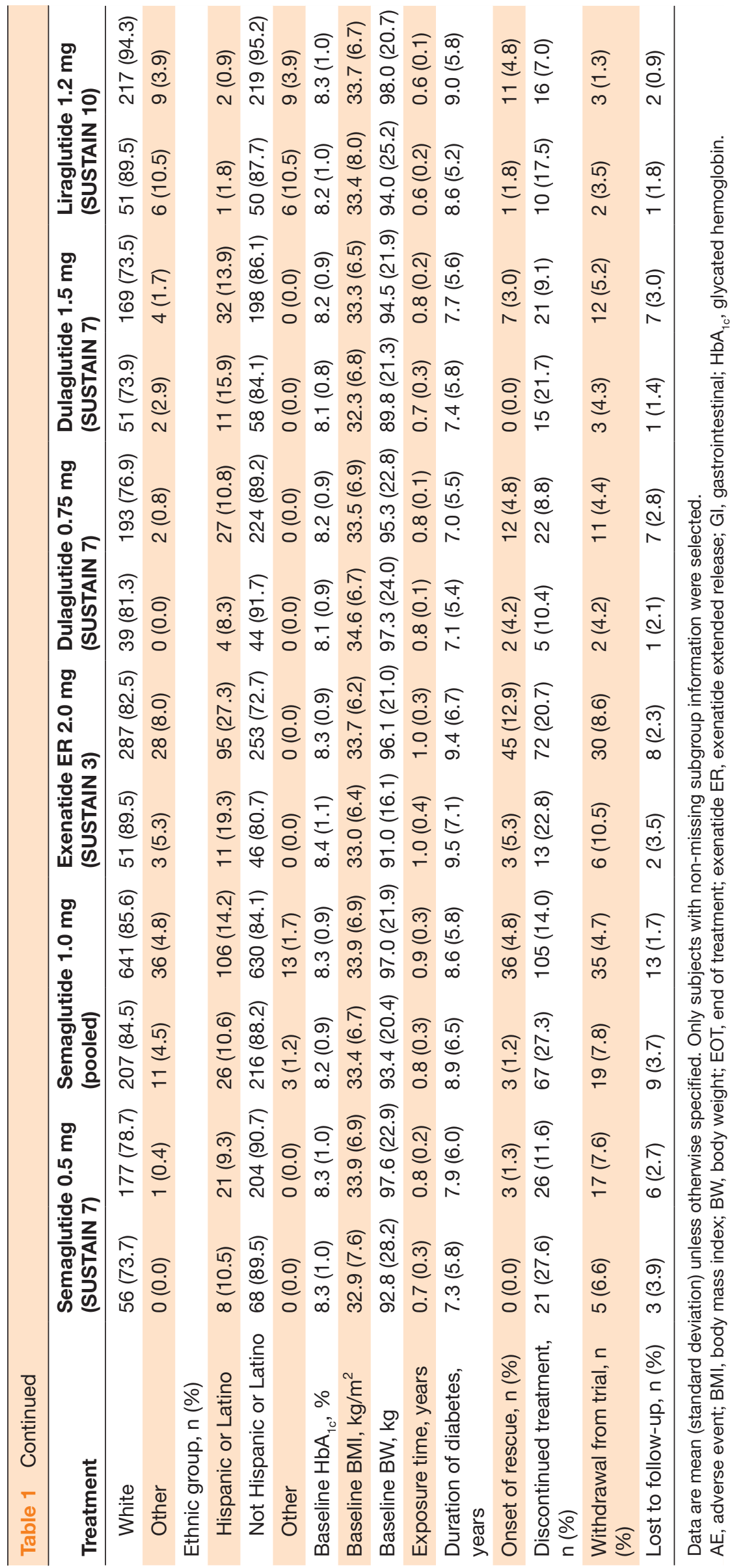



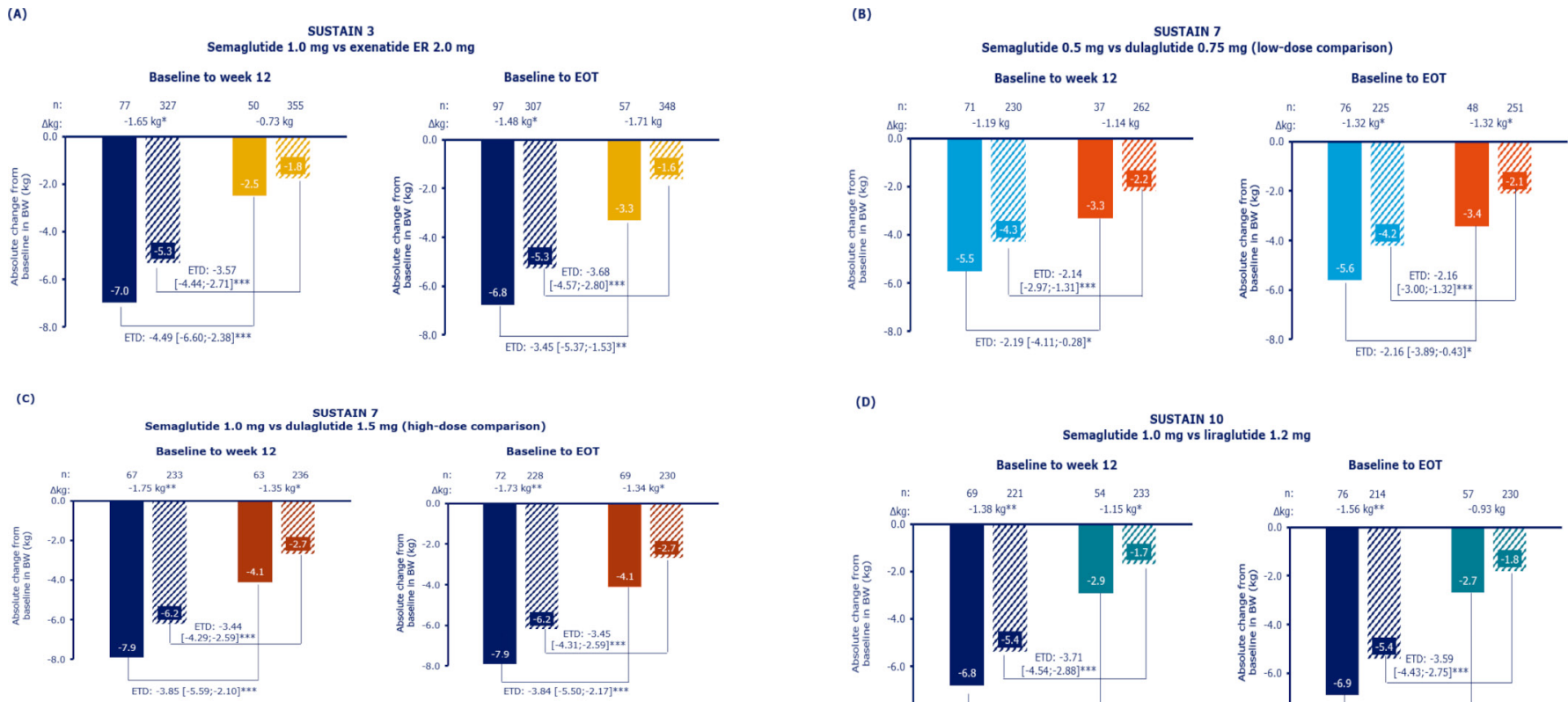

$\mathrm{kg}$ vs $-4.3 \mathrm{~kg}(\mathrm{p}=0.0542)$ for semaglutide $0.5 \mathrm{mg}$ and $-7.9 \mathrm{~kg}$ vs $-6.2 \mathrm{~kg}(\mathrm{p}=0.0074)$ for semaglutide $1.0 \mathrm{mg}$ (figure 1B,C). The corresponding values for dulaglutide $0.75 \mathrm{mg}$ and dulaglutide $1.5 \mathrm{mg}$ were $-3.3 \mathrm{~kg}$ vs

(A)

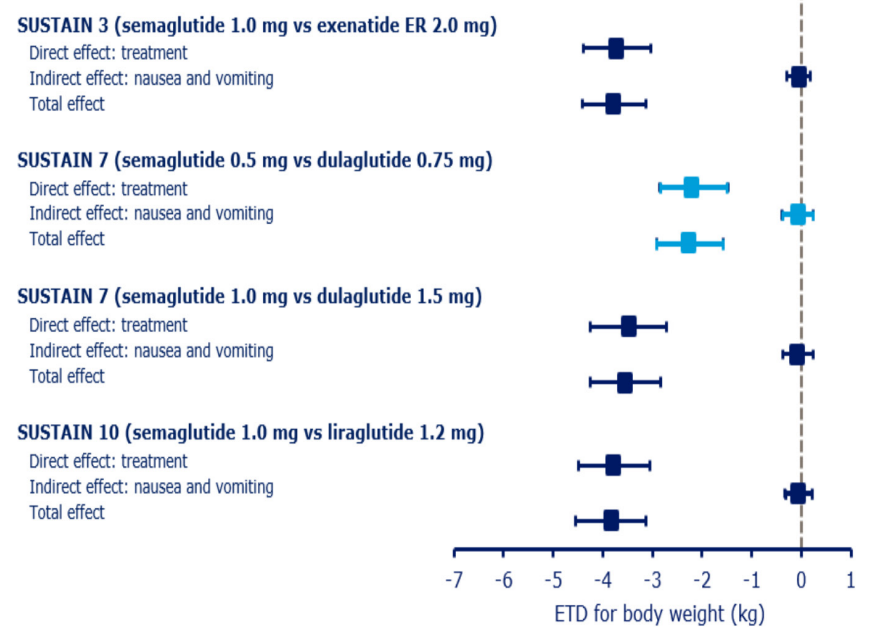

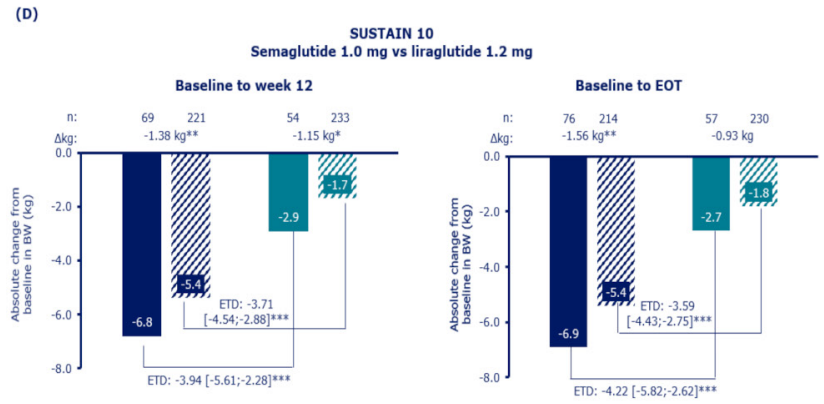

Figure 1 Absolute change from baseline in BW at EOT by nausea/vomiting occurring at any time from baseline to week 12 and at any time from baseline to EOT in SUSTAIN 3 (A), SUSTAIN 7 (B,C) and SUSTAIN 10 (D). ${ }^{*} P<0.05 ;{ }^{* \star} p<0.01 ;{ }^{* \star *} p<0.0001$. EOT was at week 56 for SUSTAIN 3, week 40 for SUSTAIN 7 and week 30 for SUSTAIN 10. Values are estimated means from a mixed model for repeated measurements analysis using 'on-treatment without rescue medication' data from subjects in the full analysis set. Values in square brackets indicate $95 \%$ Cls. BW, body weight; $\Delta \mathrm{kg}$, differences in body weight within treatment arms; EOT, end of treatment; ETD, estimated treatment difference; exenatide ER, exenatide extended release.

$-2.2 \mathrm{~kg}(\mathrm{p}=0.1153)$ and $-4.1 \mathrm{~kg}$ vs $-2.7 \mathrm{~kg}(\mathrm{p}=0.0340)$, respectively.

In subjects experiencing nausea/vomiting at any time from baseline to EOT versus those who did not experience

(B)

SUSTAIN 3 (semaglutide $1.0 \mathrm{mg}$ vs exenatide ER $2.0 \mathrm{mg}$ ) Direct effect: treatment

Indirect effect: nausea and vomiting

Total effect

SUSTAIN 7 (semaglutide $0.5 \mathrm{mg}$ vs dulaglutide $0.75 \mathrm{mg}$ ) Direct effect: treatment

Indirect effect: nausea and vomiting

Total effect

SUSTAIN 7 (semaglutide $1.0 \mathrm{mg}$ vs dulaglutide $1.5 \mathrm{mg}$ ) Direct effect: treatment

Indirect effect: nausea and vomiting

Total effect

SUSTAIN 10 (semaglutide $1.0 \mathrm{mg}$ vs liraglutide $1.2 \mathrm{mg}$ )

Direct effect: treatment

Indirect effect: nausea and vomiting

Total effect

Figure 2 Mediation analysis of direct (due to treatment) and indirect (due to nausea or vomiting) effects on weight loss for subjects treated with semaglutide from baseline to week 12 (A) and from baseline to end of treatment (B) in the SUSTAIN 3 , 7 and 10 trials. Data are 'on-treatment without rescue medication' ETDs (95\% Cls) for the change from baseline at (A) at any time in the first 12 weeks and (B) week 56 (SUSTAIN 3), week 40 (SUSTAIN 7) or week 30 (SUSTAIN 10) from all randomized patients exposed to at least one dose of trial product (full analysis set). Post-baseline data were analyzed using a mixed model for repeated measurements that included the interaction of treatment and any nausea/vomiting. ETD, estimated treatment difference; exenatide ER, exenatide extended release. 
these events, the weight change was $-5.6 \mathrm{~kg}$ vs $-4.2 \mathrm{~kg}$ at EOT $(\mathrm{p}=0.0236)$ for semaglutide $0.5 \mathrm{mg}$ and $-7.9 \mathrm{~kg}$ vs $-6.2 \mathrm{~kg}$ for semaglutide $1.0 \mathrm{mg}(\mathrm{p}=0.0051$; figure $1 \mathrm{~B}, \mathrm{C})$. The corresponding values for dulaglutide $0.75 \mathrm{mg}$ and dulaglutide $1.5 \mathrm{mg}$ were $-3.4 \mathrm{~kg}$ vs $-2.1 \mathrm{~kg}(\mathrm{p}=0.0375)$ and $-4.0 \mathrm{~kg}$ vs $-2.7 \mathrm{~kg}(\mathrm{p}=0.226)$, respectively.

ETDs (95\% CIs) favored semaglutide versus dulaglutide in all comparisons (figure 1B,C).

\section{SUSTAIN 10 (semaglutide versus liraglutide)}

At EOT, a weight change of $-6.8 \mathrm{~kg}$ was observed in subjects treated with semaglutide $1.0 \mathrm{mg}$ experiencing nausea/vomiting from randomization to week 12 vs $-5.4 \mathrm{~kg}$ in those who did not experience these events $(\mathrm{p}=0.0071)$. The corresponding values for liraglutide were -2.9 vs $-1.7 \mathrm{~kg}(\mathrm{p}=0.0295$; figure 1D).

In subjects treated with semaglutide $1.0 \mathrm{mg}$ experiencing nausea/vomiting at any time from randomization to EOT, a weight change of $-6.9 \mathrm{~kg}$ vs $-5.4 \mathrm{~kg}$ at EOT was observed versus those who did not experience these events $(\mathrm{p}=0.0021)$. The corresponding values for liraglutide were $-2.7 \mathrm{vs}-1.8 \mathrm{~kg}$ ( $\mathrm{p}=0.0528$; figure $1 \mathrm{D})$.

ETDs (95\% CIs) favored semaglutide versus liraglutide in all comparisons (figure 1D).

\section{Mediation analyses of BW reduction by nausea/vomiting SUSTAIN 3 (semaglutide versus exenatide ER)}

Mediation analyses showed that $0.05 \mathrm{~kg}$ of a total of 3.78 $\mathrm{kg}$ weight loss at EOT (week 56) observed with semaglutide versus exenatide ER in SUSTAIN 3 was mediated by nausea/vomiting from baseline to week 12 ( $p<0.0001$; figure 2A). Similarly, only $0.09 \mathrm{~kg}$ of a total of $3.78 \mathrm{~kg}$ was mediated by nausea/vomiting at any time from baseline to EOT ( $\mathrm{p}<0.0001$; figure $2 \mathrm{~B}$ ).

\section{SUSTAIN 7 (semaglutide versus dulaglutide)}

In SUSTAIN 7, $0.06 \mathrm{~kg}$ of a total of $2.26 \mathrm{~kg}$ of the greater weight loss at EOT (week 40) observed with semaglutide $0.5 \mathrm{mg}$ vs dulaglutide $0.75 \mathrm{mg}$ and $0.08 \mathrm{~kg}$ of a total of $3.55 \mathrm{~kg}$ for semaglutide $1.0 \mathrm{mg}$ vs dulaglutide $1.5 \mathrm{mg}$ was mediated by nausea/vomiting from baseline to week 12 (both $\mathrm{p}<0.0001$; figure 2A). In SUSTAIN 7, $0.03 \mathrm{~kg}$ of $2.26 \mathrm{~kg}$ of the greater weight loss at EOT observed with semaglutide $0.5 \mathrm{mg}$ vs dulaglutide $0.75 \mathrm{mg}$ and $0.04 \mathrm{~kg}$ of a total of $3.55 \mathrm{~kg}$ for semaglutide $1.0 \mathrm{mg}$ vs dulaglutide $1.5 \mathrm{mg}$ was mediated by nausea/vomiting at any time up to the EOT (both $\mathrm{p}<0.0001$; figure $2 \mathrm{~B}$ ).

\section{SUSTAIN 10 (semaglutide versus liraglutide)}

Mediation analysis showed that $0.05 \mathrm{~kg}$ of a total of 3.82 $\mathrm{kg}$ weight loss at EOT (week 30) observed with semaglutide $1.0 \mathrm{mg}$ vs liraglutide $1.2 \mathrm{mg}$ in SUSTAIN 10 was mediated by nausea/vomiting from baseline to week 12 $(p<0.0001$; figure 2A). Similarly, only $0.09 \mathrm{~kg}$ of the total of $3.82 \mathrm{~kg}$ weight loss observed with semaglutide versus liraglutide at EOT was mediated by nausea/vomiting at any time up to the EOT $(\mathrm{p}<0.0001$; figure $2 \mathrm{~B})$.

\section{DISCUSSION}

The rationale for conducting this posthoc analysis of SUSTAIN 3, 7 and 10 trials was to investigate whether GI AEs contributed to the superior weight loss observed with semaglutide versus the other GLP-1RAs, exenatide ER, dulaglutide or liraglutide. In this posthoc analysis, we found that in SUSTAIN 3, 7 and 10, subjects who experienced nausea/ vomiting, or any of the five evaluated commonly reported GI AEs, generally had slightly greater weight loss compared with subjects who did not experience these symptoms (with some exceptions). In addition, treatment with semaglutide resulted in a significantly greater weight loss than with exenatide ER, dulaglutide or liraglutide, also in subjects who did not experience nausea/vomiting, suggesting that the superior weight loss observed with semaglutide was not related to the occurrence of these events. Mediation analyses support this observation and establish that the superior weight loss seen with semaglutide (2.26 to $3.82 \mathrm{~kg}$ ) versus exenatide ER, dulaglutide or liraglutide was independent of GI AEs (only 0.03 to $0.09 \mathrm{~kg}$ due to nausea/vomiting). This is consistent with the previous analysis of the SUSTAIN $1-5$ trials, which showed that a small amount $(0.07$ to $0.5 \mathrm{~kg})$ of the total ETD (2.3 to $6.3 \mathrm{~kg}$ ) in weight loss at EOT versus mixedclass comparators was due to nausea/vomiting ${ }^{29}$ - thus, the majority of the weight-loss effect for semaglutide was not mediated by GI AEs such as nausea/vomiting.

Furthermore, in this analysis, there was no evidence of a temporal association between the incidence of GI AEs and weight loss at EOT. The prevalence of GI AEs with GLP-1RA treatment was previously found to peak within the initial 12 weeks of treatment and decline thereafter. ${ }^{33}$ However, subjects in all treatment arms experienced weight loss between baseline and week 12, and from baseline to EOT (SUSTAIN 3, week 56; SUSTAIN 7, week 40; SUSTAIN 10, week 30).

Excess weight is an important contributing factor in the complex etiology of T2D, ${ }^{4}$ and $\mathrm{BW}$ control is an important factor in the individualized management of T2D. ${ }^{467}$ GLP1RAs are established and effective therapies for T2D and can be prescribed at all stages of T2D. ${ }^{6}$ In addition to managing glucose levels, GLP-1RAs also reduce BW, ${ }^{7435}$ and this potential for weight loss has been reflected by the GLP-1RA liraglutide (3.0 $\mathrm{mg}$ once daily) gaining approval as a treatment for obesity. ${ }^{36}$ Because GI AEs including nausea, vomiting or diarrhea are the most common type of $\mathrm{AE}$ with GLP-1RAs, ${ }^{25-27}$ it is important to establish whether the weight loss difference between treatment is mediated through the occurrence of GI AEs.

The previous mediation analysis of the SUSTAIN 1-5 trials showed that only a small component of the superior weight loss with semaglutide was associated with GI AEs. ${ }^{29}$ Although GI AEs tend to be more common with semaglutide versus GLP-1RA comparators, they are usually reported during the dose-escalation phase of the trial ${ }^{38}$ and, consistent with the GLP-1RA class, are generally mild to moderate in severity and transient in nature. ${ }^{27}$

In this analysis, the fact that greater weight loss with semaglutide versus class comparators was minimally affected by 
GI AEs indicates involvement of alternative mechanisms. The unique physicochemical properties of semaglutide may contribute to the greater weight loss observed versus exenatide ER, dulaglutide or liraglutide. In a randomized controlled trial, semaglutide was associated with lower energy intake and higher BW loss versus placebo, the mechanisms likely being less appetite and food cravings, better control of eating and lower preference for fat-rich foods. ${ }^{39}$ Other GLP1RAs promote weight loss through a similar mechanism of action; ${ }^{40}$ hence, the difference between semaglutide and other GLP-1RAs may just be quantitative. Although current evidence is limited to animal studies, the data suggest that semaglutide-associated weight loss is centrally mediated through the activation of areas of the brain involved in appetite control and reward, including the hypothalamus neural circuits, the arcuate nucleus, the pro-opiomelanocortin neurons and the nucleus of the tractus solitarius. ${ }^{41-43}$

Subjects experiencing nausea/vomiting had a lower baseline BW and were more likely to discontinue treatment compared with subjects not experiencing them. Of note, despite the lower baseline BW, these subjects still experienced greater weight loss with semaglutide; this could be because semaglutide produces weight loss, irrespective of baseline BW, across a range of exposures. ${ }^{38}$

The strengths of this study are: GI AEs were analyzed in week 12, which is the time-point when they peak, as well as any time from baseline to EOT; it is an intention-to-treat analysis; mediation analysis of BW reduction was used to calculate differences between groups (not only for nausea/vomiting but also for other GI AEs); semaglutide treatment resulted in significantly greater weight loss than comparators even in subjects who did not experience GI AEs which supports the hypothesis; similar results from SUSTAIN 1-5 trials also support the hypothesis.

Potential limitations of this post hoc analysis include its inherent retrospective nature and that it was not sufficiently powered to detect the effects assessed. For example, the small number of subjects per treatment arm in the groups that experienced GI AE; therefore, results should be interpreted in this context. Another possible limitation is the different durations of follow-up for subjects with GI AE in all three trials. In addition, the results should be viewed in the context that SUSTAIN 3, 7 and 10 were open-label trials and nausea is a subjective symptom. Furthermore, in the mediation analysis, the effect of 'one unit' mediator was assumed to be the same in the treatment arms being compared. Mediation analyses rely on strong, unverifiable assumptions, and the results of the analysis may be biased in case of potential unknown confounders that affect the risk of experiencing GI AEs as well as change in BW.

\section{CONCLUSION}

In this post hoc analysis of SUSTAIN 3, 7 and 10, nausea/ vomiting contributed minimally to the significantly greater BW reductions with semaglutide versus exenatide ER, dulaglutide or liraglutide. These reductions were independent of the individual GI AEs of nausea, vomiting, diarrhea, dyspepsia and constipation in a subset of GLP-1RA class comparators with which GI AEs are the most commonly observed AEs.

\section{Author affiliations}

${ }^{1}$ Department of Internal Medicine/Endocrinology, University of Texas Southwestern

Medical Center, Dallas, Texas, USA

${ }^{2}$ Novo Nordisk A/S, Søborg, Denmark

${ }^{3}$ Clinique Ambroise Paré, Neuilly sur Seine, France

${ }^{4}$ UMRS 1138, Metabolic Inflammation in Diabetes and its Complications Cordeliers Research Centre, Paris, France

${ }^{5}$ Diabetes Division, Katholisches Klinikum Bochum, St Josef Hospital, Bochum, Germany

${ }^{6}$ Endocrinology, Millennium Physician Group, Englewood, USA

${ }^{7}$ Endocrinology and Metabolism, University of Manitoba, Winnipeg, Manitoba,

Canada

${ }^{8}$ Department of Cardiovascular and Metabolic Medicine, Institute of Life Course and Medical Sciences, University of Liverpool, Liverpool, UK

Acknowledgements We thank all the trial subjects, investigators and trial-site staff members who were involved in the SUSTAIN trials; Charlotte Hindsberger (Novo Nordisk) for reviewing the manuscript and providing statistical input and Stacy Carl-McGrath and Priya Talluri (AXON Communications funded by Novo Nordisk A/S) for medical writing and editorial assistance.

Contributors All authors contributed to the design and conduct of the trials, the analysis and interpretation of the data and the preparation, review and approval of the manuscript.

Funding This study and the associated trials were supported by Novo Nordisk A/S Denmark. Grant/award number not applicable.

Competing interests IL reports receiving grants, personal fees and non-financial support from Novo Nordisk, both for and outside the submitted work; grants from Merck and Mylan; personal fees from Valeritas, TARGET Pharma, Intarcia and Mannkind; personal fees and non-financial support from Boehringer Ingelheim, Astra Zeneca, Janssen and Eli Lilly \& Co; grants and non-financial support from Pfizer; grant, personal fees and non-financial support from Sanofi; all outside the submitted work. TH and SM do not have any conflicts of interest. MM reports receiving payment from Novo Nordisk for the submitted work; serving as consultant and on the advisory board of Novo Nordisk and Servier; consultant for Eli Lilly \& Co and serving on the advisory board of Merck Sharp \& Dohme. MAN has received fees for serving on advisory boards or lecturing for Astra Zeneca, BerlinChemie/Menarini, Boehringer Ingelheim, Eli Lilly \& Co, Genentech, GlaxoSmithKline, Medscape, Merck Sharp\&Dohme, Novo Nordisk, Sanofi-Aventis, Sun Pharma, Takeda and grant support for clinical studies from Astra Zeneca, Eli Lilly \& Co, Glaxo Smith Kline, Merck Sharp \& Dohme, Novo Nordisk and travel support for the abovementioned activities. RdIR reports receiving speaker bureau honoraria from Novo Nordisk, Sanofi-Aventis and Boehringer Ingelheim. VW reports receiving grants, personal fees and other affiliations (ie, advisory boards) from Janssen, Boehringer Ingelheim, Astra Zeneca, Lilly, Novo Nordisk and Merck. EY reports being a full-time employee and minor stockholder of Novo Nordisk. JW reports grants, personal fees and fees paid into his institution from Astra Zeneca and Novo Nordisk; fees paid into his institution from Astellas, Janssen, Lilly and Rhythm Pharmaceuticals; personal fees and fees paid into his institution from Boehringer Ingelheim, Napp, Mundipharma International; grants and personal fees from Takeda.

\section{Patient consent for publication Not required}

Provenance and peer review Not commissioned; externally peer reviewed.

Data availability statement All data relevant to the study are included in the article or uploaded as supplemental information. SUSTAIN 3: https://clinicaltrials. gov/ct2/show/NCT01885208SUSTAIN 7: https://clinicaltrials.gov/ct2/show/ NCT02648204 SUSTAIN 10: https://clinicaltrials.gov/ct2/show/NCT03191396

Open access This is an open access article distributed in accordance with the Creative Commons Attribution Non Commercial (CC BY-NC 4.0) license, which permits others to distribute, remix, adapt, build upon this work non-commercially, and license their derivative works on different terms, provided the original work is properly cited, appropriate credit is given, any changes made indicated, and the use is non-commercial. See: http://creativecommons.org/licenses/by-nc/4.0/.

\section{ORCID iDs}

Ildiko Lingvay http://orcid.org/0000-0001-7006-7401

John Wilding http://orcid.org/0000-0003-2839-8404 


\section{REFERENCES}

1 International Diabetes Federation. Canada country report 2017 \& 2045, 2017. Available: https://reports.instantatlas.com/report/view/ 704ee0e6475b4af885051bcec15f0e2c/CAN

2 Public Health England. Adult obesity and type 2 diabetes, 2014. Available: https://assets.publishing.service.gov.uk/government/ uploads/system/uploads/attachment_data/file/338934/Adult_ obesity_and_type_2_diabetes_.pdf

3 World Health Organization. Obesity and overweight fact sheet, 2003. Available: https://www.who.int/dietphysicalactivity/media/en/gsfs_ obesity.pdf

4 Wilding JPH. The importance of weight management in type 2 diabetes mellitus. Int J Clin Pract 2014;68:682-91.

5 Wing RR, Lang W, Wadden TA, et al. Benefits of modest weight loss in improving cardiovascular risk factors in overweight and obese individuals with type 2 diabetes. Diabetes Care 2011;34:1481-6.

6 Davies MJ, D'Alessio DA, Fradkin J, et al. Management of hyperglycaemia in type 2 diabetes, 2018. A consensus report by the American Diabetes Association (ADA) and the European Association for the study of Diabetes (EASD). Diabetologia 2018;61:2461-98.

7 American Diabetes Association. Obesity management for the treatment of type 2 diabetes: standards of medical care in diabetes-2020. Diabetes Care 2020;43:S89-97.

8 Buse JB, Wexler DJ, Tsapas A, et al. Update to: management of hyperglycaemia in type 2 diabetes, 2018. A consensus report by the American diabetes association (ADA) and the European association for the study of diabetes (EASD). Diabetologia 2019;2020:221-8.

9 Nauck MA, Meier JJ, Cavender MA, et al. Cardiovascular actions and clinical outcomes with glucagon-like peptide-1 receptor agonists and dipeptidyl peptidase-4 inhibitors. Circulation 2017:136:849-70

10 Madsbad S. Review of head-to-head comparisons of glucagon-like peptide-1 receptor agonists. Diabetes Obes Metab 2016;18:317-32.

11 Trujillo JM, Nuffer W, Ellis SL. Glp-1 receptor agonists: a review of head-to-head clinical studies. Ther Adv Endocrinol Metab 2015;6:19-28.

12 Nauck MA, Meier JJ. Management of endocrine disease: are all GLP-1 agonists equal in the treatment of type 2 diabetes? Eur $J$ Endocrinol 2019;181:R211-34.

13 Novo Nordisk. Ozempic $₫$ (semaglutide) prescribing information, 2020. Available: https://www.novo-pi.com/ozempic.pdf

14 Novo Nordisk. Rybelsus $®$ (semaglutide) prescribing information. Available: https://www.novo-pi.com/rybelsus.pdf

15 Sorli C, Harashima S-I, Tsoukas GM, et al. Efficacy and safety of once-weekly semaglutide monotherapy versus placebo in patients with type 2 diabetes (SUSTAIN 1): a double-blind, randomised, placebo-controlled, parallel-group, multinational, multicentre phase 3 A trial. Lancet Diabetes Endocrinol 2017;5:251-60.

16 Ahrén B, Masmiquel L, Kumar H, et al. Efficacy and safety of once-weekly semaglutide versus once-daily sitagliptin as an add-on to metformin, thiazolidinediones, or both, in patients with type 2 diabetes (SUSTAIN 2): a 56-week, double-blind, phase 3A, randomised trial. Lancet Diabetes Endocrinol 2017;5:341-54.

17 Ahmann AJ, Capehorn M, Charpentier G, et al. Efficacy and safety of once-weekly semaglutide versus exenatide ER in subjects with type 2 diabetes (SUSTAIN 3): a 56-week, open-label, randomized clinical trial. Diabetes Care 2018;41:258-66.

18 Aroda VR, Bain SC, Cariou B, et al. Efficacy and safety of onceweekly semaglutide versus once-daily insulin glargine as add-on to metformin (with or without sulfonylureas) in insulin-naive patients with type 2 diabetes (SUSTAIN 4): a randomised, open-label, parallel-group, multicentre, multinational, phase $3 \mathrm{~A}$ trial. Lancet Diabetes Endocrinol 2017;5:355-66.

19 Rodbard HW, Lingvay I, Reed J, et al. Semaglutide added to basal insulin in type 2 diabetes (SUSTAIN 5): a randomized, controlled trial. $J$ Clin Endocrinol Metab 2018:103:2291-301.

20 Marso SP, Bain SC, Consoli A, et al. Semaglutide and cardiovascular outcomes in patients with type 2 diabetes. N Engl J Med 2016;375:1834-44.

21 Pratley RE, Aroda VR, Lingvay I, et al. Semaglutide versus dulaglutide once weekly in patients with type 2 diabetes (SUSTAIN 7): a randomised, open-label, phase 3B trial. Lancet Diabetes Endocrinol 2018;6:275-86.

22 Lingvay I, Catarig A-M, Frias JP, et al. Efficacy and safety of once-weekly semaglutide versus daily canagliflozin as add-on to metformin in patients with type 2 diabetes (SUSTAIN 8): a doubleblind, phase 3B, randomised controlled trial. Lancet Diabetes Endocrinol 2019;7:834-44.

23 Zinman B, Bhosekar V, Busch R, et al. Semaglutide once weekly as add-on to SGLT-2 inhibitor therapy in type 2 diabetes (SUSTAIN 9): a randomised, placebo-controlled trial. Lancet Diabetes Endocrinol 2019;7:356-67.

24 Capehorn MS, Catarig A-M, Furberg JK, et al. Efficacy and safety of once-weekly semaglutide $1.0 \mathrm{mg}$ vs once-daily liraglutide 1.2 $\mathrm{mg}$ as add-on to 1-3 oral antidiabetic drugs in subjects with type 2 diabetes (SUSTAIN10). Diabetes Metab 2020;46:100-9.

25 Bettge K, Kahle M, Abd El Aziz MS, et al. Occurrence of nausea, vomiting and diarrhoea reported as adverse events in clinical trials studying glucagon-like peptide- 1 receptor agonists: a systematic analysis of published clinical trials. Diabetes Obes Metab 2017;19:336-47.

26 Raccah D. Safety and tolerability of glucagon-like peptide-1 receptor agonists: unresolved and emerging issues. Expert Opin Drug Saf 2017;16:227-36

27 Horowitz M, Aroda VR, Han J, et al. Upper and/or lower gastrointestinal adverse events with glucagon-like peptidereceptor agonists: incidence and consequences. Diabetes Obes Metab 2017:19:672-81.

28 Novo Nordisk. Data on file

29 Ahrén B, Atkin SL, Charpentier G, et al. Semaglutide induces weight loss in subjects with type 2 diabetes regardless of baseline BMI or gastrointestinal adverse events in the SUSTAIN 1 to 5 trials. Diabetes Obes Metab 2018;20:2210-9.

30 AstraZeneca. Byetta $($ (exenatide) prescribing information, 2015. Available: http://www.azpicentral.com/byetta/pi_byetta.pdf

31 Jendle J, Grunberger G, Blevins T, et al. Efficacy and safety of dulaglutide in the treatment of type 2 diabetes: a comprehensive review of the dulaglutide clinical data focusing on the AWARD phase 3 clinical trial program. Diabetes Metab Res Rev 2016;32:776-90.

32 Vansteelandt S, Bekaert M, Lange T. Imputation strategies for the estimation of natural direct and indirect effects. Epidemiol Methods 2012;1:131-58

33 Nauck MA, Petrie JR, Sesti G, et al. A phase 2, randomized, dose-finding study of the novel once-weekly human GLP-1 analog, semaglutide, compared with placebo and open-label liraglutide in patients with type 2 diabetes. Diabetes Care 2016;39:231-41.

34 Abd El Aziz MS, Kahle M, Meier JJ, et al. A meta-analysis comparing clinical effects of short- or long-acting GLP-1 receptor agonists versus insulin treatment from head-to-head studies in type 2 diabetic patients. Diabetes Obes Metab 2017;19:216-27.

35 Potts JE, Gray LJ, Brady EM, et al. The effect of glucagon-like peptide 1 receptor agonists on weight loss in type 2 diabetes: a systematic review and mixed treatment comparison meta-analysis. PLoS One 2015;10:e0126769.

36 European Medicines Agency. Saxenda (liraglutide) summary of product characteristics, 2018. Available: https://www.ema.europa. eu/en/documents/product-information/saxenda-epar-productinformation_en.pdf

37 Novo Nordisk. Saxenda $($ (liraglutide) prescribing information, March 2020. Available: https://www.novo-pi.com/saxenda.pdf

38 Petri KCC, Ingwersen SH, Flint A, et al. Exposure-response analysis for evaluation of semaglutide dose levels in type 2 diabetes. Diabetes Obes Metab 2018;20:2238-45.

39 Blundell J, Finlayson G, Axelsen M, et al. Effects of once-weekly semaglutide on appetite, energy intake, control of eating, food preference and body weight in subjects with obesity. Diabetes Obes Metab 2017;19:1242-51.

40 Sposito AC, Berwanger O, de Carvalho LSF, et al. GLP-1RAs in type 2 diabetes: mechanisms that underlie cardiovascular effects and overview of cardiovascular outcome data. Cardiovasc Diabetol 2018;17:157.

41 Jensen CB, Pyke C, Rasch MG, et al. Characterization of the glucagon-like peptide-1 receptor in male mouse brain using a novel antibody and in situ hybridization. Endocrinology 2018;159:665-75.

42 Müller TD, Finan B, Bloom SR, et al. Glucagon-Like peptide 1 (GLP1). Mol Metab 2019;30:72-130.

43 Gabery S, Salinas CG, Paulsen SJ, et al. Semaglutide lowers body weight in rodents via distributed neural pathways. JCl Insight 2020;5:133429 